KYUNGPOOK Math. J. 49(2009), 313-319

\title{
On Local Properties of Factored Fourier Series
}

\section{HüSEYİN BOR}

Department of Mathematics, Erciyes University, 38039 Kayseri, Turkey

e-mail : bor@erciyes.edu.tr

ABSTRACT. In the present paper, a theorem dealing with local property of $\left|\bar{N}, p_{n}, \theta_{n}\right|_{k}$ summability of factored Fourier series which generalizes a result of Mazhar [8], has been proved. Some new results have also been obtained.

\section{Introduction}

Let $\sum a_{n}$ be a given infinite series with partial sums $\left(s_{n}\right)$. We denote by $t_{n}$ the $\mathrm{n}$-th $(\mathrm{C}, 1)$ mean of the sequence $\left(n a_{n}\right)$. A series $\sum a_{n}$ is said to be summable $|C, 1|_{k}, k \geq 1$, if (see [6])

$$
\sum_{n=1}^{\infty} \frac{1}{n}\left|t_{n}\right|^{k}<\infty
$$

Let $\left(p_{n}\right)$ be a sequence of positive numbers such that

$$
P_{n}=\sum_{v=0}^{n} p_{v} \rightarrow \infty \quad \text { as } \quad n \rightarrow \infty, \quad\left(P_{-i}=p_{-i}=0, i \geq 1\right)
$$

The sequence-to-sequence transformation

$$
\sigma_{n}=\frac{1}{P_{n}} \sum_{v=0}^{n} p_{v} s_{v}
$$

defines the sequence $\left(\sigma_{n}\right)$ of the Riesz mean or simply the $\left(\bar{N}, p_{n}\right)$ mean of the sequence $\left(s_{n}\right)$, generated by the sequence of coefficients $\left(p_{n}\right)$ (see [7]). The series $\sum a_{n}$ is said to be summable $\left|\bar{N}, p_{n}\right|_{k}, k \geq 1$, if (see [2])

$$
\sum_{n=1}^{\infty}\left(P_{n} / p_{n}\right)^{k-1}\left|\Delta \sigma_{n-1}\right|^{k}<\infty \text {, }
$$

where

$$
\Delta \sigma_{n-1}=-\frac{p_{n}}{P_{n} P_{n-1}} \sum_{v=1}^{n} P_{v-1} a_{v}, \quad n \geq 1 .
$$

Received May 29, 2008; accepted September 3, 2008.

2000 Mathematics Subject Classification: 40G99, 42A24, 42B24.

Key words and phrases: absolute summability, infinite series, local property, Fourier series. 
In the special case $p_{n}=1$ for all values of $\mathrm{n},\left|\bar{N}, p_{n}\right|_{k}$ summability is the same as $|C, 1|_{k}$ summability. Also, if we take $k=1$ and $p_{n}=1 /(n+1)$, then summability $\left|\bar{N}, p_{n}\right|_{k}$ is equivalent to the summability $|R, \log n, 1|$. Let $\left(\theta_{n}\right)$ be any sequence of positive constants. The series $\sum a_{n}$ is said to be summable $\left|\bar{N}, p_{n}, \theta_{n}\right|_{k}, k \geq 1$, if (see [12])

$$
\sum_{n=1}^{\infty} \theta_{n}^{k-1}\left|\Delta \sigma_{n-1}\right|^{k}<\infty
$$

If we take $\theta_{n}=\frac{P_{n}}{p_{n}}$, then $\left|\bar{N}, p_{n}, \theta_{n}\right|_{k}$ summability reduces to $\left|\bar{N}, p_{n}\right|_{k}$ summability. Also, if we take $\theta_{n}=n$ and $p_{n}=1$ for all values of $n$, then we get $|C, 1|_{k}$ summability.

Furthermore, if we take $\theta_{n}=n$, then $\left|\bar{N}, p_{n}, \theta_{n}\right|_{k}$ summability reduces to $\left|R, p_{n}\right|_{k}$ (see [4]) summability. A sequence $\left(\lambda_{n}\right)$ is said to be convex if $\Delta^{2} \lambda_{n} \geq 0$ for every positive integer $n$, where $\Delta^{2} \lambda_{n}=\Delta\left(\Delta \lambda_{n}\right)$ and $\Delta \lambda_{n}=\lambda_{n}-\lambda_{n+1}$.

Let $f(t)$ be a periodic function with period $2 \pi$ and integrable $(L)$ over $(-\pi, \pi)$. Without any loss of generality we may assume that the constant term in the Fourier series of $f(t)$ is zero, so that

$$
\int_{-\pi}^{\pi} f(t) d t=0
$$

and

$$
f(t) \sim \sum_{n=1}^{\infty}\left(a_{n} \cos n t+b_{n} \sin n t\right)=\sum_{n=1}^{\infty} A_{n}(t) .
$$

It is well known (see [13]) that the convergence of the Fourier series at $t=x$ is a local property of the generating function $\mathrm{f}(\mathrm{t})$ (i.e., it depends only on the behaviour of $\mathrm{f}$ in a arbitrarily small neighbourhood of $x$ ), and hence the summability of the Fourier series at $t=x$ by any regular linear summability method is also a local property of the generating function $\mathrm{f}(\mathrm{t})$.

\section{Known result}

Mohanty [11] has demonstrated that the summability $|R, \log n, 1|$ of

$$
\sum A_{n}(t) / \log (n+1)
$$

at $t=x$, is a local property of the generating function of $\sum A_{n}(t)$. Later on Matsumoto [9] improved this result by replacing the series (9) by

$$
\sum A_{n}(t) / \log \log (n+1)^{1+\epsilon}, \epsilon>0 .
$$

Generalizing the above result Bhatt [1] proved the following theorem. 
Theorem A. If $\left(\lambda_{n}\right)$ is a convex sequence such that $\sum n^{-1} \lambda_{n}$ is convergent, then the summability $|R, \log n, 1|$ of the series $\sum A_{n}(t) \lambda_{n} \log n$ at a point can be ensured by a local property.

Mishra [10] has also proved the following most general theorem dealing with local property.

Theorem B. If $\left(p_{n}\right)$ is a sequence such that

$$
\begin{aligned}
P_{n} & =O\left(n p_{n}\right), \\
P_{n} \Delta p_{n} & =O\left(p_{n} p_{n+1}\right),
\end{aligned}
$$

then the summability $\left|\bar{N}, p_{n}\right|$ of the series

$$
\sum_{n=1}^{\infty} A_{n}(t) \lambda_{n} P_{n} / n p_{n}
$$

at a point can be ensured by local property, where $\left(\lambda_{n}\right)$ is as in Theorem $A$.

On the other hand, Bor [3] has extended Theorem B and he proved that under the conditions of Theorem B the result also holds for the summability $\left|\bar{N}, p_{n}\right|_{k}$, $k \geq 1$. Later on, he [5] has further generalized his result in the following way :

Theorem C. Let $k \geq 1,\left(p_{n}\right)$ and $\left(\lambda_{n}\right)$ be a sequences such that

$$
\begin{aligned}
& \Delta X_{n}=O(1 / n), \quad X_{n}=\frac{P_{n}}{n p_{n}}, \\
& \sum_{n=1}^{\infty} X_{n}^{k-1} \frac{\left|\lambda_{n}\right|^{k}+\left|\lambda_{n+1}\right|^{k}}{n}<\infty, \\
& \sum_{n=1}^{\infty}\left(X_{n}^{k}+1\right)\left|\Delta \lambda_{n}\right|<\infty .
\end{aligned}
$$

Then the summability $\left|\bar{N}, p_{n}\right|_{k}$ of the series $\sum A_{n}(t) \lambda_{n} X_{n}$ at a point can be ensured by local property.

Mazhar [8] has generated Theorem C in the following form.

Theorem D. Let $k \geq 1,\left(p_{n}\right)$ and $\left(\lambda_{n}\right)$ be sequences such that

$$
\begin{aligned}
& \Delta\left(P_{n-1} X_{n}\right)=O\left(\frac{P_{n}}{n}\right), \quad X_{n}=\frac{P_{n}}{n p_{n}}, \\
& \sum_{n=1}^{\infty} X_{n}^{k-1} \frac{\left|\lambda_{n}\right|^{k}}{n}<\infty, \\
& \sum_{n=1}^{\infty} X_{n+1}\left|\Delta \lambda_{n}\right|<\infty .
\end{aligned}
$$


Then the summability $\left|\bar{N}, p_{n}\right|_{k}$ of the series $\sum A_{n}(t) \lambda_{n} X_{n}$ at point can be ensured by a local property.

\section{The main result}

The aim of this paper is to generalize Theorem $\mathrm{D}$ for $\left|\bar{N}, p_{n}, \theta_{n}\right|_{k}$ summability. That is, we shall prove the following theorem.

Theorem. Let $k \geq 1,\left(p_{n}\right)$ and $\left(\lambda_{n}\right)$ be sequences such that conditions (17), (19) are satisfied. If $\left(\frac{\theta_{n} p_{n}}{P_{n}}\right)$ is a non-decreasing sequence and

$$
\sum_{n=1}^{\infty} \theta_{n}^{k-1}\left(\frac{p_{n}}{P_{n}}\right)^{k}\left|\lambda_{n}\right|^{k} X_{n}^{k}<\infty
$$

then the summability $\left|\bar{N}, p_{n}, \theta_{n}\right|_{k}$ of the series $\sum A_{n}(t) \lambda_{n} X_{n}$ at a point can be ensured by a local property.

It should be noted that if we take $\theta_{n}=\frac{P_{n}}{p_{n}}$, then we get Theorem D. In this case condition (20) reduces to condition (18). In fact

$$
\begin{aligned}
\sum_{n=1}^{\infty} \theta_{n}^{k-1}\left(\frac{p_{n}}{P_{n}}\right)^{k}\left|\lambda_{n}\right|^{k} X_{n}^{k} & =\sum_{n=1}^{\infty}\left(\frac{P_{n}}{p_{n}}\right)^{k} \frac{p_{n}}{P_{n}}\left(\frac{p_{n}}{P_{n}}\right)^{k}\left|\lambda_{n}\right|^{k} X_{n}^{k} \\
& =\sum_{n=1}^{\infty} X_{n}^{k} \frac{p_{n}}{P_{n}}\left|\lambda_{n}\right|^{k} \\
& =\sum_{n=1}^{\infty} X_{n}^{k} \frac{1}{n X_{n}}\left|\lambda_{n}\right|^{k} \\
& =\sum_{n=1}^{\infty} X_{n}^{k-1} \frac{\left|\lambda_{n}\right|^{k}}{n}<\infty .
\end{aligned}
$$

Proof of theorem. Since the behaviour of the Fourier series, as far as convergence is concerned, for a particular value of $x$ depends on the behaviour of the function in the immediate neighbourhood of this point only and hence to complete the proof of the theorem it is sufficent to prove that if $\left(s_{n}\right)$ is bounded, then under the conditions of our theorem $\sum a_{n} \lambda_{n} X_{n}$ is summable $\left|\bar{N}, p_{n}, \theta_{n}\right|_{k}, k \geq 1$.

Let $\left(T_{n}\right)$ denotes the $\left(\bar{N}, p_{n}\right)$ mean of the series $\sum a_{n} \lambda_{n} X_{n}$. Then, by definition, we have

$$
T_{n}=\frac{1}{P_{n}} \sum_{v=0}^{n} p_{v} \sum_{r=0}^{v} a_{r} \lambda_{r} X_{r}=\frac{1}{P_{n}} \sum_{v=0}^{n}\left(P_{n}-P_{v-1}\right) a_{v} \lambda_{v} X_{v} .
$$


Then, we have

$$
T_{n}-T_{n-1}=\frac{p_{n}}{P_{n} P_{n-1}} \sum_{v=1}^{n} P_{v-1} a_{v} \lambda_{v} X_{v}, \quad n \geq 1, \quad\left(P_{-1}=0\right) .
$$

Now using Abel's transformation, we have

$$
\begin{aligned}
& T_{n}-T_{n-1} \\
= & \frac{p_{n}}{P_{n} P_{n-1}} \sum_{v=1}^{n-1} s_{v} \lambda_{v} \Delta\left(P_{v-1} X_{v}\right)+\frac{p_{n} s_{n} \lambda_{n} X_{n}}{P_{n}} \\
= & \frac{p_{n}}{P_{n} P_{n-1}} \sum_{v=1}^{n-1} s_{v} \lambda_{v} \Delta\left(P_{v-1} X_{v}\right)+\frac{p_{n}}{P_{n} P_{n-1}} \sum_{v=1}^{n-1} s_{v} P_{v} X_{v+1} \Delta \lambda_{v}+\frac{p_{n} s_{n} \lambda_{n} X_{n}}{P_{n}} \\
= & T_{n, 1}+T_{n, 2}+T_{n, 3}, \quad \text { say. }
\end{aligned}
$$

To complete the proof of the Theorem, by Minkowski's inequality for $k>1$, it is sufficient to show that

$$
\sum_{n=1}^{\infty} \theta_{n}^{k-1}\left|T_{n, r}\right|^{k}<\infty, \quad \text { for } \quad r=1,2,3
$$

Now applying Hölder's inequality, we have that

$$
\begin{aligned}
& \sum_{n=2}^{m+1} \theta_{n}^{k-1}\left|T_{n, 1}\right|^{k} \\
\leq & \sum_{n=2}^{m+1} \theta_{n}^{k-1}\left(\frac{p_{n}}{P_{n}}\right)^{k} \frac{1}{P_{n-1}^{k}}\left|\sum_{v=1}^{n-1} s_{v} \lambda_{v} \Delta\left(P_{v-1} X_{v}\right)\right|^{k} \\
= & O(1) \sum_{n=2}^{m+1} \theta_{n}^{k-1}\left(\frac{p_{n}}{P_{n}}\right)^{k} \frac{1}{P_{n-1}^{k}}\left\{\sum_{v=1}^{n-1} \frac{\left|\lambda_{v}\right| P_{v}}{v}\right\}^{k} \\
= & O(1) \sum_{n=2}^{m+1} \theta_{n}^{k-1}\left(\frac{p_{n}}{P_{n}}\right)^{k} \frac{1}{P_{n-1}^{k}}\left\{\sum_{v=1}^{n-1}\left|\lambda_{v}\right| X_{v} p_{v}\right\}^{k} \\
= & O(1) \sum_{n=2}^{m+1} \theta_{n}^{k-1}\left(\frac{p_{n}}{P_{n}}\right)^{k} \frac{1}{P_{n-1}} \sum_{v=1}^{n-1} p_{v}\left|\lambda_{v}\right|^{k} X_{v}^{k} \times\left\{\frac{1}{P_{n-1}} \sum_{v=1}^{n-1} p_{v}\right\}^{k-1} \\
= & \sum_{v=1}^{m} p_{v}\left|\lambda_{v}\right|^{k} X_{v}^{k} \sum_{n=v+1}^{m+1}\left(\frac{\theta_{n} p_{n}}{P_{n}}\right)^{k-1} \frac{p_{n}}{P_{n} P_{n-1}} \\
= & O(1) \sum_{v=1}^{m} p_{v}\left|\lambda_{v}\right|^{k} X_{v}^{k}\left(\frac{\theta_{v} p_{v}}{P_{v}}\right)^{k-1} \frac{\sum_{n=v+1}^{m+1}}{P_{n} P_{n-1}}
\end{aligned}
$$




$$
\begin{aligned}
& =O(1) \sum_{v=1}^{m} p_{v}\left|\lambda_{v}\right|^{k} X_{v}^{k} \theta_{v}^{k-1}\left(\frac{p_{v}}{P_{v}}\right)^{k-1} \frac{1}{P_{v}} \\
& =O(1) \sum_{v=1}^{m} \theta_{v}^{k-1}\left(\frac{p_{v}}{P_{v}}\right)^{k}\left|\lambda_{v}\right|^{k} X_{v}^{k}=O(1) \quad \text { as } \quad m \rightarrow \infty
\end{aligned}
$$

by virtue of the hypotheses of the Theorem. Again

$$
\begin{aligned}
& \sum_{n=2}^{m+1} \theta_{n}^{k-1}\left|T_{n, 2}\right|^{k} \\
\leq & \sum_{n=2}^{m+1} \theta_{n}^{k-1}\left(\frac{p_{n}}{P_{n}}\right)^{k} \frac{1}{P_{n-1}^{k}}\left\{\sum_{v=1}^{n-1}\left|s_{v}\right| P_{v} X_{v+1}\left|\Delta \lambda_{v}\right|\right\}^{k} \times\left\{\sum_{v=1}^{n-1} X_{v+1}\left|\Delta \lambda_{v}\right|\right\}^{k-1} \\
= & O(1) \sum_{v=1}^{n-1} P_{v}^{k} X_{v+1}\left|\Delta \lambda_{v}\right| \sum_{n=v+1}^{m+1}\left(\frac{\theta_{n} p_{n}}{P_{n}}\right)^{k} \frac{p_{n}}{P_{n} P_{n-1}^{k}} \\
= & O(1) \sum_{v=1}^{m} P_{v}^{k} X_{v+1}\left|\Delta \lambda_{v}\right|\left(\frac{\theta_{v} p_{v}}{P_{v}}\right)^{k-1} \frac{1}{P_{v}^{k-1}} \sum_{n=v+1}^{m+1} \frac{p_{n}}{P_{n} P_{n-1}} \\
= & O(1) \sum_{v=1}^{m} P_{v}^{k} X_{v+1}\left|\Delta \lambda_{v}\right|\left(\frac{\theta_{v} p_{v}}{P_{v}}\right)^{k-1} \frac{1}{P_{v}^{k-1}} \frac{1}{P_{v}} \\
= & O(1)\left(\frac{\theta_{1} p_{1}}{P_{1}}\right)^{k-1} \sum_{v=1}^{m} X_{v+1}\left|\Delta \lambda_{v}\right| \\
= & O(1) \sum_{v=1}^{m} X_{v+1}\left|\Delta \lambda_{v}\right|=O(1) \quad \text { as } m \rightarrow \infty
\end{aligned}
$$

in view of the hypotheses of the Theorem.

Finally, we have that

$$
\begin{aligned}
\sum_{n=1}^{m} \theta_{n}^{k-1}\left|T_{n, 3}\right|^{k} & =\sum_{n=1}^{m} \theta_{n}^{k-1}\left|\frac{p_{n} s_{n} \lambda_{n} X_{n}}{P_{n}}\right|^{k} \\
& =O(1) \sum_{n=1}^{m} \theta_{n}^{k-1}\left(\frac{p_{n}}{P_{n}}\right)^{k}\left|\lambda_{n}\right|^{k} X_{n}^{k}=O(1) \quad \text { as } \quad m \rightarrow \infty,
\end{aligned}
$$

by virtue of the hypotheses of the Theorem. Therefore we get that

$$
\sum_{n=1}^{m} \theta_{n}^{k-1}\left|T_{n, r}\right|^{k}=O(1) \quad \text { as } \quad m \rightarrow \infty, \quad \text { for } \quad r=1,2,3 .
$$

which completes the proof of the Theorem. If we take $\theta_{n}=n$ and $p_{n}=1$ for all values of $n$, then we get a new result for $|C, 1|_{k}$ summability. Also, if we take 
$\theta_{n}=n$, then we have another new result for $\left|R, p_{n}\right|_{k}$ summablity.

\section{References}

[1] S. N. Bhatt, An aspect of local property of $|R, \log n, 1|$ summability of the factored Fourier series, Proc. Nat. Inst. India, 26(1960), 69-73.

[2] H. Bor, On two summability methods, Math. Proc. Cambridge Philos. Soc., 97(1985), 147-149.

[3] H. Bor, Local property of $\left|\bar{N}, p_{n}\right|_{k}$ summability of factored Fourier series, Bull. Inst. Math. Acad. Sinica, 17(1989), 165-170.

[4] H. Bor, On the relative strength of two absolute summability methods, Proc. Amer. Math. Soc., 113(1991), 1009-1012.

[5] H. Bor, On the local property of $\left|\bar{N}, p_{n}\right|_{k}$ summability of factored Fourier series, J. Math. Anal. Appl., 163(1992), 220-226.

[6] T. M. Flett, On an extension of absolute summability and some theorems of Littlewood and Paley, Proc. London Math. Soc., 7(1957), 113-141.

[7] G. H. Hardy, Divergent series, Oxford Univ. Press, Oxford, 1949.

[8] S.M. Mazhar, A note on the localization of $\left|\bar{N}, p_{n}\right|_{k}$ summability of Fourier series, Kumamoto J. Math., 12(1999), 1-8.

[9] K. Matsumoto, Local property of the summability $\left|R, \lambda_{n}, 1\right|$, Tohoku Math. J., 8(2)(1956), 114-124.

[10] K. N. Mishra, Multipliers for $\left|\bar{N}, p_{n}\right|$ summability of Fourier series, Bull. Inst. Math. Acad. Sinica, 14(1986), 431-438.

[11] R. Mohanty, On the summability $|R, \log w, 1|$ of Fourier series, J. London Math. Soc., 25(1950), 67-72.

[12] W. T. Sulaiman, On some summability factors of infinite series, Proc. Amer. Math. Soc., 115(1992), 313-317.

[13] E.C. Titchmarsh, The Theory of Functions, Oxford Univ. Press, London, 1961. 